\title{
REVIEW
}

\section{Outcome parameters in spinal cord injury research: a systematic review using the International Classification of Functioning, Disability and Health (ICF) as a reference}

\author{
MWM Post ${ }^{1}$, I Kirchberger ${ }^{2}$, M Scheuringer ${ }^{3,5}$, MM Wollaars ${ }^{1}$ and S Geyh ${ }^{4}$ \\ ${ }^{1}$ Rehabilitation Centre 'De Hoogstraat' and Rudolf Magnus Institute for Neuroscience, Utrecht, The Netherlands; ${ }^{2}$ ICF Research \\ Branch of the WHO FIC CC (DIMDI), IHRS, Department of Physical Medicine and Rehabilitation, Ludwig-Maximilian University, \\ Munich, Germany; ${ }^{3}$ ICF Research Branch of the WHO FIC CC (DIMDI), IHRS, Department of Physical Medicine and Rehabilitation, \\ Ludwig-Maximilian University, Munich, Germany and ${ }^{4}$ Swiss Paraplegic Research, Nottwil, Switzerland
}

\begin{abstract}
Objectives: This study is part of the development of an International Classification of Functioning, Disability and Health (ICF) Core Set for spinal cord injury (SCI). Its specific objectives were to identify outcome parameters reported in published studies on individuals with $\mathrm{SCl}$ in the early post-acute and chronic situation, and to identify and quantify the concepts of the reported parameters using the ICF as a reference.

Methods: Electronic searches of Medline, EMBASE, PsycINFO and CINAHL from 2001 to 2005 were carried out. All outcome parameters and their underlying concepts were retrieved from the included studies. These concepts were linked to categories of the ICF using standardized rules.

Results: From the 6681 abstracts retrieved, 2205 were randomly selected (33.0\%) and 281 studies met the inclusion criteria (12.7\%). A total number of 5217 concepts were retrieved from standardized and non-standardized measures, of which 4049 (77.6\%) could be linked to 175 different ICF categories: 56 out of 114 Body Functions, 19 out of 56 Body Structures, 62 out of 118 Activities and Participation and 38 out of 74 Environmental Factors categories. Second-level categories reported in $>20 \%$ of all studies were pain, remunerative employment, health services, systems and policies, school education and higher education.

Conclusion: The ICF provides a valuable reference to identify and quantify the concepts of measures focusing on $\mathrm{SCl}$ in the early post-acute and chronic situation. The findings show a great diversity in the consequences of $\mathrm{SCl}$ and underscore the importance of social participation and environment for people with $\mathrm{SCl}$.

Spinal Cord (2010) 48, 522-528; doi:10.1038/sc.2009.177; published online 5 January 2010
\end{abstract}

Keywords: mesh:spinal cord; systematic literature review; rehabilitation, outcome assessment nonmesh: ICF; ICF Core Set

\section{Introduction}

Spinal cord injury (SCI) is multifaceted and may involve all body functions below the level of the neurological lesion. ${ }^{1}$ The typical spectrum of activity limitations and participation restrictions relate to mobility and self-care, ${ }^{2,3}$ work, maintaining social relationships, participating in leisure activities and being active members of the community. ${ }^{4}$ Activity limitations and participation restrictions are highly dependent on environmental factors such as accessibility and availability of adaptive equipment and support. ${ }^{5,6}$

Correspondence: Dr MWM Post, De Hoogstraat, Rembrandtkade 10, Utrecht, 3583 TM, The Netherlands.

E-mail: m.post@dehoogstraat.nl

${ }^{5}$ Current address: Outcomes Research, MSD Sharp \& Dohme GmbH, Haar, Germany.

Received 2 June 2009; revised 30 October 2009; accepted 1 November 2009; published online 5 January 2010
The International Classification of Functioning, Disability and Health $(\mathrm{ICF})^{7}$ may serve as a comprehensive and universally accepted framework to classify and describe functioning, disability and health in people with all kinds of diseases or conditions, including SCI. The ICF comprises four components: Body Functions, Body Structures, Activities and Participation and Environmental Factors. ${ }^{7}$ The ICF is structured hierarchically in chapters and categories of different levels. To give an example, the third-level ICF category, 'b7353, Tone of muscles of lower half of body' is one element of the second-level category, 'b735, Muscle tone functions', which is in turn an element of the chapter 'b7, Neuromusculoskeletal and movement-related functions', which is part of the ICF component 'b, Body Functions'.

Completeness and exhaustive detail are essential traits of an international language of functioning and disability; but 
not every user of the ICF will require the range and detail that ICF provides. Clinicians, for example, who wish to apply the bio-psycho-social model to their daily practice, will require only a fraction of health and health-related states classified in the ICF. ICF Core Sets are selections of categories out of the ICF that are relevant for people with a specific condition or in a specific setting. ${ }^{8}$ Comprising Core Sets of ICF categories for specific conditions may provide guidance for clinical assessment, assignment of clinical interventions and selection of measures for evaluation. Furthermore, ICF Core Sets can contribute to the comparability of clinical data between patients, institutions and countries, and provide a basis to improve communication among professionals and between settings. ${ }^{8}$ To date, ICF Core Sets have been developed for various chronic conditions. However, in SCI different contexts have to be taken into account. As an 'ICF Core Set for neurological conditions in the acute context' was already developed, ${ }^{9}$ the project aimed at developing ICF Core Sets for SCI for both the early post-acute and chronic contexts. $^{10}$

The development process of the ICF Core Sets for SCI is divided into a preparatory phase and a consensus conference. ${ }^{10}$ The preparatory phase was meant to consider the perspectives of the patients, experts and the researchers in the development process and to identify those aspects of functioning and health that are relevant in their view. ${ }^{10}$ The researcher perspective is addressed with the systematic review described in this article. The consumer perspective is addressed both in a quantitative and structured way in the empirical study ${ }^{11}$ and in a qualitative way using focus groups. ${ }^{12}$ Finally, the expert opinion, involving professionals from different backgrounds, is addressed with the expert survey. ${ }^{13}$ As the ICF Core Sets are to be used in clinical as well as research settings, it is important to ensure that these perspectives have been taken into account in the development process. One method of capturing the researchers' perspective and the topics that are relevant in their work is to systematically review the outcomes measured and reported in SCI research. This requires first the identification of outcome parameters reported in published studies, the linkage of the concepts contained in these parameters to the ICF and the quantitative analyses of the ICF categories that represent the parameters. ${ }^{14,15}$ The results show what ICF categories are often addressed in research and are thereby important from the researchers' point of view. These categories are candidates for inclusion in the ICF Core Set for SCI. ${ }^{10}$

The objective of this systematic review is, first, to identify outcome parameters reported in published studies focusing on individuals with spinal cord injury (SCI) in the early postacute and chronic situation and, second, to identify and quantify the concepts of the reported parameters using the ICF as a reference.

\section{Materials and methods}

The review was performed in three steps. First, searches in the literature database were conducted. Second, outcome parameters were extracted from the included studies, and third, the concepts within the outcome parameters were linked to the ICF.

\section{Search strategy and inclusion criteria}

We aimed to include studies providing empirical data on adults with SCI, because the context of children is different from the context of adults and might better be addressed in a separate core set. Further, we aimed to include all types and etiologies of SCI to provide an unbiased review of SCI research. Electronic searches in CINAHL, Medline, Embase and PsycINFO were performed. We first selected studies published in English between 2001 and 2005, using the search terms spinal cord, parapleg*, tetrapleg* and quadripleg*. The detailed search strategies are available upon request. Because the number of retrieved abstracts turned out to be very large, a random sample of 33\% was drawn using the random sample procedure in SPSS (SPSS Inc., Chicago, IL, USA) and only these abstracts were checked for inclusion.

From this random sample, studies were included according to the following criteria:

(1) Describing subjects with SCI only. SCI was defined as impairment or loss of motor and/or sensory function in the cervical, thoracic, lumbar or sacral segments of the spinal cord secondary to damage of neural elements within the spinal canal. ${ }^{16}$ This includes complete and incomplete SCI, all neurological levels and traumatic and non-traumatic causes. (2) Describing >10 subjects, and excluding case studies, reviews, meta-analyses, ecological studies and study protocols, (3) including only subjects who are at least 18 years of age at the study entry, and excluding studies on children and adolescents with SCI, (4) including only subjects who had an SCI of onset within 14 days, and excluding studies describing individuals with degenerative disorders as Guillain-Barré syndrome or genetically predisposed diseases, and (5) including only subjects who were in the early post-acute or chronic situation. The early postacute situation begins with active rehabilitation and ends with the completion of the first comprehensive rehabilitation after the acute SCI. The chronic situation follows the early post-acute situation. This working definition was based on a worldwide consensus of researchers involved in the data collection and was approved by the steering committee of the project to develop the ICF Core Sets for Spinal Cord Injury.

Finally, we excluded studies that fitted all inclusion criteria that focused on co-morbidities, such as, for example, the presence of traumatic brain injury in people with SCI.

\section{Data extraction procedure}

Two researchers extracted characteristics of the randomly selected studies and all study outcome parameters reported in these studies using a standardized electronic data record form. In this study a parameter was defined as a measured or documented construct that was reported as a study outcome. Parameters comprised standardized as well as non-standardized measures. The single items of standardized questionnaires and observational measures were regarded as single 
parameters. If necessary, questionnaires were obtained by reference checking, searches in books on clinical measures, contacting the author or Internet searches. After data extraction of the parameters, both researchers compared their results. Initial disagreement could be solved after discussion between the two researchers (MP and MW). If there was disagreement after this, a third person was consulted (MS). Second, the underlying concepts of the parameters were specified. A concept was defined as one separate meaningful entity. One or several concepts could be derived from a single parameter. For example, from the single item or parameter 'how severe was your pain during regular daily work', two concepts were derived: 'pain' and 'daily work'.

\section{Linking to the ICF}

The concepts of the retrieved parameters were then linked to ICF categories using specified linking rules. ${ }^{14,15}$ These rules were developed to standardize the linking process. Linking rules are, for example, 'each concept is linked to the most precise ICF category' and 'do not use the so-called 'unspecified' categories of the ICF but the lower level (more general) category'. A specific rule for questionnaires is, for example, 'the response options of an item are linked if they contain concepts'. A specific linking rule for technical and clinical measures is 'define the aim with which the intervention was applied in the concrete investigation in form of a concept' ${ }^{\prime 15}$ For example, the previously mentioned concepts 'pain' and 'daily work' were linked to the concepts, 'b280, Pain' and 'd850, Remunerative employment'. Pulse rate was, for example, linked to 'b4550, General physical endurance' if the aim was to measure exercise tolerance. If a concept was too general to allow a decision on the linking to a specific ICF category, the concept was considered as 'not defined'. Examples include activities of daily living, general health or quality of life. If a concept pertained to personal factors, the code 'personal factor' was attributed as personal factors are not listed in the ICF. Examples include coping with pain, self-esteem or religion. If a concept described an aspect of functioning and health that is not covered by the ICF, the code 'not covered' was attributed. Examples are survival or rest. If a concept pertained to a diagnosis or disease, the code 'health condition' was attributed. Examples include pneumonia, urinary tract infection and cancer. Basic demographic and SCI characteristics of patients, such as age and level of injury, were not linked but used to describe the characteristics of the included studies.

\section{Quality assurance}

A peer-review process was conducted for determining the inter-rater reliability of abstract checking, data extraction and linking. In the abstract checking phase, one reviewer reviewed all 2205 abstracts for eligibility. A second reviewer independently reviewed every 25 th abstract $(N=88)$. Agreement about inclusion or exclusion was observed for 82 (93.4\%) of these abstracts. The remaining six articles were agreed upon inclusion or exclusion after discussion. All 281 included articles were examined by two reviewers for parameter extraction. In $78.5 \%$ articles $(N=219)$, there was total agreement. Finally, the linking was performed by two reviewers for 22 of the 126 questionnaires and for 270 of the 2203 parameters derived from non-standardized measures and other reported outcomes. Cohen's $\kappa$ was $0.80(95 \%$ confidence interval $0.75-0.83$ ) and 0.55 (95\% confidence interval 0.42-0.67), respectively.

\section{Data analysis}

Frequencies of the outcome measures and the linked ICF categories were reported along with percentages, relative to the total number of concepts or the total number of studies. The 95\% confidence intervals of these percentages were reported as we used a random sample of all the articles identified in the literature search. If an ICF category was assigned repeatedly in a study, it was counted only once to avoid bias. All ICF categories that referred to concepts measured in $>5 \%$ of the studies were reported. If a concept was linked to a third- or a fourth-level category, the corresponding second-level category is reported. This is appropriate, because the lower-level categories share the attributes of the corresponding higher-level categories. ${ }^{15}$

\section{Results}

The literature database searches resulted in 6681 unique abstracts, of which 2205 (33\%) were randomly selected.

Table 1 Study characteristics $(N=281)$

\begin{tabular}{lr}
\hline Study type & \\
Observational & $60.1 \%$ \\
Cross-sectional & $48.8 \%$ \\
Longitudinal & $11.4 \%$ \\
Intervention & $32.4 \%$ \\
RCT & $4.3 \%$ \\
CCT & $7.5 \%$ \\
Other intervention & $20.6 \%$ \\
Other studies & $7.5 \%$ \\
& \\
Situation & \\
Early post-acute & $10.6 \%$ \\
Chronic & $56.4 \%$ \\
Mixed & $33.0 \%$ \\
& \\
Etiology & \\
Traumatic & $24.8 \%$ \\
Non-traumatic & $18.8 \%$ \\
Mixed & $17.4 \%$ \\
Unspecified & $39.0 \%$ \\
& \\
Level of SCl & \\
Tetraplegia & $17.7 \%$ \\
Paraplegia & $10.3 \%$ \\
Mixed & $62.4 \%$ \\
Unspecified & $9.6 \%$ \\
Completeness of SCl & \\
Complete & \\
Incomplete & $7.1 \%$ \\
Mixed & $10.3 \%$ \\
Unspecified & $52.1 \%$ \\
\hline Abrevions cCT & $30.5 \%$ \\
\hline
\end{tabular}

Abbreviations: $\mathrm{CCT}$, controlled clinical trial; $\mathrm{RCT}$, randomized clinical trial; $\mathrm{SCl}$, spinal cord injury. 
Table 2 Relative frequency of first-level categories of the ICF linked to the concepts contained in the parameters of 281 studies

\begin{tabular}{|c|c|c|c|}
\hline ICF chapters & Description & Number of studies & $\%(95 \% \mathrm{Cl})$ \\
\hline \multicolumn{4}{|l|}{ Body functions } \\
\hline b1 & Mental functions & 53 & $18.9(14.7-23.8)$ \\
\hline b2 & Sensory functions and pain & 99 & $35.2(29.9-41.0)$ \\
\hline b4 & $\begin{array}{l}\text { Functions of the cardiovascular, hematological, immunological and } \\
\text { respiratory systems }\end{array}$ & 67 & $23.8(19.2-29.2)$ \\
\hline b5 & Functions of the digestive, metabolic and endocrine systems & 50 & $17.8(13.8-22.7)$ \\
\hline b6 & Genitourinary and reproductive functions & 74 & $26.3(21.5-31.8)$ \\
\hline b7 & Neuromusculoskeletal and movement-related functions & 90 & $32.0(26.8-37.7)$ \\
\hline b8 & Functions of the skin and related functions & 37 & $13.2(9.7-17.6)$ \\
\hline \multicolumn{4}{|l|}{ Body structures } \\
\hline s1 & Nervous system & 37 & $13.2(9.7-17.6)$ \\
\hline s7 & Movement-related & 47 & $16.7(12.8-21.5)$ \\
\hline \multicolumn{4}{|c|}{ Activities and participation } \\
\hline $\mathrm{d} 4$ & Mobility & 93 & $33.1(27.9-38.8)$ \\
\hline d5 & Self-care & 111 & $39.5(34.0-45.3)$ \\
\hline d6 & Domestic activities & 25 & $8.9(6.1-12.8)$ \\
\hline d7 & Interpersonal interactions and relationships & 31 & $11.0(7.9-15.2)$ \\
\hline $\mathrm{d} 8$ & Major life areas & 93 & $33.1(27.9-38.8)$ \\
\hline d9 & Community, social and civic life & 36 & $12.8(9.4-17.2)$ \\
\hline \multicolumn{4}{|l|}{ Environmental factors } \\
\hline e1 & Products and technology & 115 & $40.9(35.3-46.8)$ \\
\hline e3 & Support and relationships & 48 & $17.1(13.1-21.9)$ \\
\hline e5 & Services, systems and policies & 79 & $28.1(23.2-33.6)$ \\
\hline Personal factors & & 124 & $44.0(38.2-50.2)$ \\
\hline Not covered & & 151 & $53.5(47.7-59.7)$ \\
\hline $\begin{array}{l}\text { Not defined-health } \\
\text { condition }\end{array}$ & & 167 & $59.2(53.4-65.2)$ \\
\hline Not defined_-other & & 118 & $41.8(36.2-48.0)$ \\
\hline
\end{tabular}

Abbreviations: $\mathrm{Cl}$, confidence interval; ICF, International Classification of Functioning, Disability and Health.

Note that only categories measured in $\geqslant 5 \%$ of all studies are displayed.

A total of 281 studies (12.7\%) met the inclusion criteria. Most studies had an observational design and concerned the chronic phase of SCI. The number of subjects ranged from 11 to 7981 (median $=47$; interquartile range 22-139). Mean age ranged from 25 to 83 (median $=43.0$; interquartile range 38.6-56.0) and the percentage of females ranged from 0 to $100 \% \quad$ (median $=22.7$; interquartile range $13.8-33.3 \%$ ). Other study characteristics are shown in Table 1.

A total of 146 different standardized questionnaires and observational measures were retrieved, of which $126(86.3 \%)$ could be obtained and were linked. Most often used measures were the FIM (functional independence measure)/ motor-FIM (27 studies), Satisfaction with Life scale (14 studies), Craig Handicap and Reporting Technique (13 studies), and the Japanese Orthopaedic Association scale (12 studies).

A total of 5217 concepts were extracted from the parameters identified in the 281 studies. Out of these, 355 concepts (6.8\%) were considered as 'not defined', 270 (5.2\%) as 'not covered', $222(4.2 \%)$ as 'health condition' and 321 $(6.2 \%)$ as 'personal factors'. The other 4049 concepts could be linked to a total of 401 different ICF categories. In all, 13 concepts $(0.3 \%)$ were linked to an ICF component, 261 concepts (5.0\%) were linked to the first level of the ICF, 1903 concepts $(36.5 \%)$ to second-level ICF categories, 1681 concepts $(32.2 \%)$ to third-level ICF categories and 191 $(3.7 \%)$ to fourth-level ICF categories. These higher-level categories were then merged into the corresponding secondlevel categories.

In Table 2, the first-level categories, or chapters, are shown that were addressed in $>5 \%$ of all studies. This includes 7 out of 8 'Body Functions' chapters, 2 out of 7 'Body Structure' chapters, 6 out of 9 'Activities and Participation' chapters and 3 out of 5 'Environmental Factors' chapters.

Summarizing the second, third and fourth level-categories at the second level, the 4049 concepts could be linked to 175 different second-level categories: 56 out of $114(49 \%)$ to 'Body Functions' categories, 19 out of 56 (34\%) to 'Body Structures' categories, 62 out of 118 (52\%) to 'Activities and Participation' categories and 38 out of 74 (51\%) to 'Environmental Factors' categories. A total of 47 second-level categories reflected concepts measured in at least 5\% of all studies. 'B280, Pain', 'b730, Muscle power functions' and 'b620, Urinary functions' were the most often found second-level categories of the 'Body Functions' component (Table 3). 'Body Structure' categories were less often found and only 's120, Structures of the spinal cord' and 's760, Trunk' were found in $>5 \%$ of all studies (Table 4). 'D820, School education', 'd830, Higher education' and 'd850, Remunerative "employment" were the most often found second-level categories from the 'Activities and Participation' component (Table 5) and 'e580 Health services, systems and policies' was the most often found 'Environmental Factor' (Table 6). 
Table 3 Relative frequency of second-level categories of the ICF linked to the concepts contained in the parameters of 281 studies: component Body Functions

\begin{tabular}{|c|c|c|c|}
\hline ICF code & Description & Count & $\%(95 \% \mathrm{Cl})$ \\
\hline \multicolumn{4}{|c|}{ Chapter 1: mental functions (22) } \\
\hline b134 & Sleep functions & 20 & $7.1(4.7-10.7)$ \\
\hline b152 & Emotional functions & 33 & $11.7(8.5-16.0)$ \\
\hline \multicolumn{4}{|c|}{ Chapter 2: sensory functions and pain (18) } \\
\hline b270 & Sensory functions related to temperature and other stimuli & 44 & $15.7(11.9-20.4)$ \\
\hline b280 & Pain & 81 & $28.8(23.8-34.4)$ \\
\hline \multicolumn{4}{|c|}{ Chapter 4: functions of the cardiovascular, hematological, immunological and respiratory systems (16) } \\
\hline b410 & Heart functions & 20 & $7.1(4.7-10.7)$ \\
\hline b415 & Blood vessel functions & 15 & $5.3(3.3-8.6)$ \\
\hline b420 & Blood pressure functions & 16 & $5.7(3.5-9.0)$ \\
\hline b440 & Respiration functions & 26 & $9.3(6.4-13.2)$ \\
\hline b455 & Exercise tolerance functions & 16 & $5.7(3.5-9.0)$ \\
\hline \multicolumn{4}{|c|}{ Chapter 5: functions of the digestive, metabolic and endocrine systems (14) } \\
\hline b525 & Defecation functions & 39 & $13.8(10.3-18.4)$ \\
\hline \multicolumn{4}{|c|}{ Chapter 6: genitourinary and reproductive functions (11) } \\
\hline b610 & Urinary excretory functions & 38 & $13.5(10.0-18.0)$ \\
\hline b620 & Urinary functions & 56 & $19.9(15.7-25.0)$ \\
\hline b640 & Sexual functions & 19 & $6.8(4.4-10.3)$ \\
\hline \multicolumn{4}{|c|}{ Chapter 7: neuromusculoskeletal and movement-related functions (17) } \\
\hline b710 & Mobility of joint functions & 26 & $9.3(6.4-13.2)$ \\
\hline b730 & Muscle power functions & 59 & $21.1(16.6-26.1)$ \\
\hline b735 & Muscle tone functions & 29 & $10.3(7.3-14.4)$ \\
\hline b750 & Motor reflex functions & 15 & $5.3(3.3-8.6)$ \\
\hline \multicolumn{4}{|c|}{ Chapter 8: functions of the skin and related structures } \\
\hline b810 & Protective functions of the skin & 35 & $12.5(9.1-16.8)$ \\
\hline
\end{tabular}

Abbreviations: $\mathrm{Cl}$, confidence interval; ICF, International Classification of Functioning, Disability and Health.

Note that only codes measured in $\geqslant 5 \%$ of all studies are displayed.

Table 4 Relative frequency of second-level categories of the ICF linked to the concepts contained in the parameters of 281 studies: component Body Structures

\begin{tabular}{lcc}
\hline ICF code $\quad$ Description & Count & $\%(95 \%$ Cl) \\
\hline $\begin{array}{l}\text { Chapter 1: structures of the nervous system (7) } \\
\text { s120 Spinal cord and related structures }\end{array}$ & 35 & $12.5(9.1-16.8)$ \\
$\begin{array}{l}\text { Chapter 7: structures related to movement (9) } \\
\text { S760 Structure of trunk }\end{array}$ & 31 & $11.0(7.9-15.2)$ \\
\hline
\end{tabular}

Abbreviations: $\mathrm{Cl}$, confidence interval; ICF, International Classification of Functioning, Disability and Health.

Note that only codes measured in $\geqslant 5 \%$ of all studies are displayed.

\section{Discussion}

Using the ICF as a reference, it was possible to identify and quantify concepts of parameters cited in published studies on individuals with SCI. By extracting and linking all outcome parameters reported in a representative sample of 281 studies, a comprehensive and systematic overview of concepts measured in SCI research was provided. Our results thereby identify the ICF categories that, from the researchers' perspective, would be most relevant to be included in an ICF Core Set for SCI.

Elaborating on earlier systematic reviews that were part of the other ICF Core Set development projects, we extracted and linked all concepts measured in the included studies and not only the concepts that were extracted from standardized measures and clinical tests. ${ }^{17-21}$ Second, we included observational studies in addition to clinical trials in our review. Only one earlier review also included observational studies. $^{22}$

The large number of 175 different second-level categories found in this study underscores the heterogeneity of consequences of SCI in patients' functioning. However, only 23 were frequently measured (in $>10 \%$ of all studies); nine of these belonged to the component 'Body Functions', 2 to 'Body Structures', 7 to 'Activities and Participation' and 5 to 'Environmental factors'. The most-often reported secondlevel categories ( $>20 \%$ of all studies) were 'b280, Pain', 'd850, Remunerative employment', 'e580, Health services, 
systems and policies', 'd820, School education' and 'd830, Higher education'.

Categories referring to body structures and referring to mental and cognitive activities (d1-d3) were rarely the topic of SCI research. There was an interesting difference between

Table 5 Relative frequency of second-level categories of the ICF linked to the concepts contained in the parameters of 281 studies: component Activities and Participation

\begin{tabular}{|c|c|c|c|}
\hline ICF code & Description & Count & $\%(95 \% \mathrm{Cl})$ \\
\hline \multicolumn{4}{|c|}{ Chapter 4: mobility (20) } \\
\hline $\mathrm{d} 410$ & Changing basic body position & 15 & $5.3(3.3-8.6)$ \\
\hline d4415 & Maintaining a body position & 20 & $7.1(4.7-10.7)$ \\
\hline d445 & Hand and arm use & 15 & $5.3(3.3-8.6)$ \\
\hline d450 & Walking & 39 & $13.9(10.3-18.4)$ \\
\hline d455 & Moving around & 16 & $5.7(3.5-9.0)$ \\
\hline d460 & Moving around in different locations & 16 & $5.7(3.5-9.0)$ \\
\hline d465 & Moving around using equipment & 15 & $5.3(3.3-8.6)$ \\
\hline \multicolumn{4}{|c|}{ Chapter 5: self-care (9) } \\
\hline d510 & Washing oneself & 19 & $6.8(4.4-10.3)$ \\
\hline d520 & Caring for body parts & 20 & $7.1(4.7-10.7)$ \\
\hline d530 & Toileting & 43 & $15.3(11.6-20.0)$ \\
\hline d540 & Dressing & 22 & $7.8(5.2-11.6)$ \\
\hline d550 & Eating & 31 & $11.0(7.9-15.2)$ \\
\hline d570 & Looking after one's health & 22 & $7.8(5.2-11.6)$ \\
\hline \multicolumn{4}{|c|}{ Chapter 6: domestic life (9) } \\
\hline d640 & Doing housework & 16 & $5.7(3.5-9.0)$ \\
\hline \multicolumn{4}{|c|}{ Chapter 7: interpersonal interactions and relationships (11) } \\
\hline d760 & Family relationships & 17 & $6.0(3.8-9.5)$ \\
\hline d770 & Intimate relationships & 23 & $8.2(5.5-12.0)$ \\
\hline \multicolumn{4}{|c|}{ Chapter 8: major life areas (17) } \\
\hline d820 & School education & 58 & $20.6(16.3-25.8)$ \\
\hline d830 & Higher education & 58 & $20.6(16.3-25.8)$ \\
\hline d850 & Remunerative employment & 78 & $27.8(22.8-33.3)$ \\
\hline \multicolumn{4}{|c|}{ Chapter 9: community, social and civic life (7) } \\
\hline d920 & Recreation and leisure & 35 & $12.5(9.1-16.8)$ \\
\hline
\end{tabular}

Abbreviations: $\mathrm{Cl}$, confidence interval; ICF, International Classification of Functioning, Disability and Health.

Note that only codes measured in $\geqslant 5 \%$ of all studies are displayed. the relative frequencies of first-level and of second-level categories. The relative frequencies of the Mobility (d4) and Self-care (d5) chapters were high, but their corresponding second-level categories were not. Regarding mobility, only one second-level category, walking, was cited in $>10 \%$ of all studies. This is in part due to the large number (20) of different second-level categories related to mobility.

A mean of 18.6 concepts per study were retrieved. In most of the studies reviewed, one or more concepts were reported that could not be linked to the ICF, because they referred to health conditions, which are coded by the ICD-10, or to personal factors that are not classified in the ICF, or because they were not specified in enough detail to allow linking. However, the large majority of concepts found (77.8\%) could be linked, confirming the usefulness of the classification. A total of $6.2 \%$ of all concepts referred to personal factors. Personal factors is a component of the ICF, but, unfortunately, a classification of personal factors is still to be developed.

The high relative frequencies of categories referring to social participation and environmental factors not only underscore the importance of these concepts for SCI patients, but are also a reflection of the type of studies included in this review. Most of these focused on the chronic phase. An earlier review reporting on patients undergoing early post-acute rehabilitation did not reveal relative frequencies $>5 \%$ for these categories. ${ }^{22} \mathrm{~A}$ review of concepts measured in stroke trials ${ }^{21}$ found remunerative employment to be documented in $11 \%$ of all studies, and education in $<10 \%$.

Compared with earlier reviews, ${ }^{17-21}$ we found low relative frequencies of the linked ICF categories. One explanation is that these other reviews included clinical trials only, whereas we selected a much broader range of studies. As, for example, the Barthel Index is used in $>50 \%$ of all clinical stroke trials, a review including only clinical stroke trials will reveal relative frequencies $>50 \%$ for all ICF categories related to concepts extracted from the Barthel Index. ${ }^{21}$ Methodological differences have to be taken into account when comparing the results from different studies.

Table 6 Relative frequency of second-level categories of the ICF linked to the concepts contained in the parameters of 281 studies: component Environmental Factors

\begin{tabular}{|c|c|c|c|}
\hline ICF code & Description & Count & $\%(95 \% \mathrm{Cl})$ \\
\hline \multicolumn{4}{|c|}{ Chapter 1: products and technology } \\
\hline e110 & Products or substances for personal consumption & 28 & $10.0(7.0-14.0)$ \\
\hline e115 & Products and technology for personal use in daily living & 37 & $13.2(9.7-17.6)$ \\
\hline e120 & $\begin{array}{l}\text { Products and technology for personal indoor and outdoor mobility and } \\
\text { transportation }\end{array}$ & 42 & $14.9(11.3-19.6)$ \\
\hline e165 & Assets & 38 & $13.5(10.0-18.0)$ \\
\hline \multicolumn{4}{|c|}{ Chapter 3: support and relationships } \\
\hline e310 & Immediate family & 22 & $7.8(5.2-11.6)$ \\
\hline e340 & Personal care providers and personal assistants & 16 & $5.7(3.5-9.0)$ \\
\hline \multicolumn{4}{|c|}{ Chapter 5: services, systems and policies } \\
\hline e580 & Health services, systems and policies & 69 & $24.6(19.9-29.9)$ \\
\hline
\end{tabular}

Abbreviations: $\mathrm{Cl}$, confidence interval; ICF, International Classification of Functioning, Disability and Health.

Note that only codes measured in $\geqslant 5 \%$ of all studies are displayed. 
Our study further showed that cross-sectional and observational studies are most frequent in SCI research, and that intervention studies are sparse. There is a need for more longitudinal studies that might reveal changes in the medical and living conditions of people with SCI and for intervention studies to increase the evidence base of SCI rehabilitation.

\section{Limitations}

Our selection of studies might have been biased, by including only studies published in English and standardized measures available in English. Our search concerned 20012005 and it is possible that including more recent studies would have revealed different results. Furthermore, we only retrieved a random sample of $33 \%$ of the eligible studies. The confidence intervals reported however show the relatively small uncertainty associated with our estimations of relative frequencies of ICF categories. Further, linking to the ICF was not always straightforward and required frequent discussion, but the quality assurance data showed acceptable reliability of the data extraction and linking process.

\section{Conflict of interest}

The authors declare no conflict of interest.

\section{Acknowledgements}

This study was funded by the Swiss Paraplegic Research, Nottwil, Switzerland.

\section{References}

1 Vernon L, Cardenal DL, Cutter NC, Frost FS, Hammond MC, Lindblom LB et al. Spinal Cord Medicine: Principles and Practice. Demos Publication: New York, NY, 2003.

2 Gerhart K, Bergstrom E, Charlifue SW, Menter R, Whiteneck GG. Long-term spinal cord injury: functional changes over time. Arch Phys Med Rehab 1993; 74: 1030-1034.

3 Bode RK, Heineman AW. Course of functional improvement after stroke, spinal cord injury and traumatic brain injury. Arch Phys Med Rehabil 2002; 84: 100-106.

4 Noreau L, Fougeyrollas P, Post M, Asano M. Participation after spinal cord injury: the evolution of conceptualization and measurement. J Neurol Phys Ther 2005; 29: 147-156.

5 Whiteneck GG, Meade MA, Dijkers M, Tate DG, Bushnik T, Forchheimer MB. Environmental factors and their role in participation and life satisfaction after spinal cord injury. Arch Phys Med Rehab 2004; 85: 1793-1803.

6 Biering-Sörensen F, Hansen RB, Biering-Sörensen J. Mobility aids and transport possibilities 10-45 years after spinal cord injury. Spinal Cord 2004; 42: 699-706.

7 World Health Organization. International Classification of Functioning, Disability and Health: ICF. WHO: Geneva, 2001.

8 Stucki G, Grimby G. Applying the ICF in medicine. J Rehabil Med 2004; 44S: 5-6.
9 Ewert T, Grill E, Bartholomeyczik S, Finger M, Mokkrusch T, Kostanjsek $\mathrm{N}$ et al. ICF Core Sets for patients with neurological conditions in the acute hospital. Disabil Rehabil 2005; 27: 367-373.

10 Biering-Sörensen F, Scheuringer M, Baumberger M, Charlifue SW, Post MW, Montero F et al. Developing core sets for persons with spinal cord injury based on the International Classification of Functioning, Disability and Health as a way to specify functioning. Spinal Cord 2006; 44: 541-546.

11 Scheuringer M, Kirchberger I, Boldt C, Hoogland-Eriks I, Rauch A, Velstra I-M et al. Identification of problems in individuals with spinal cord injury from the health professional perspective using the ICF: a worldwide expert survey. Spinal Cord (in press).

12 Kirchberger I, Sinnott A, Charlifue S, Kovindha A, Lüthi H, Campbell $\mathrm{R}$ et al. Problems in individuals with spinal cord injury from the consumer perspective: a worldwide qualitative study using the ICF. Spinal Cord (submitted).

13 Kirchberger I, Biering-Sørensen F, Charlifue S, Baumberger M, Campbell R, Kovindha A et al. Identification of the most common problems in functioning of individuals with spinal cord injury using the International Classification of Functioning, Disability and Health. Spinal Cord (e-pub ahead of print 15 September 2009).

14 Cieza A, Brockow T, Ewert T, Amman E, Kollerits B, Chatterji S et al. Linking health-status measurements to the International Classification of Functioning, Disability and Health. J Rehab Med 2002; 34: 205-210.

15 Cieza A, Geyh S, Chatterji S, Kostanjsek N, stün B, Stucki G. ICF linking rules: an update based on lessons learned. J Rehabil Med 2005; 37: 212-218.

16 Marino RJ, Barros T, Biering-Sorensen F, Burns SP, Donovan WH, Graves DE et al. International standards for neurological classification of spinal cord injury. J Spinal Cord Med 2003; 26(Suppl 1): S50-S56.

17 Brockow T, Cieza A, Kuhlow H, Sigl T, Franke T, Harder M et al. Identifying the concepts contained in outcome measures of clinical trials on musculoskeletal disorders and chronic widespread pain using the International Classification of functioning, Disability and Health as a reference. J Rehabil Med 2004; 44(Suppl): 30-36.

18 Wolff B, Cieza A, Parentin A, Rauch A, Sigl T, Brockow T et al. Identifying the concepts contained in outcome measures of clinical trials on four internal disorders using the International Classification of functioning, Disability and Health as a reference. J Rehabil Med 2004; 44(Suppl): 37-42.

19 Brockow T, Duddeck K, Geyh S, Schwarzkopf SR, Weigl M, Franke $\mathrm{T}$ et al. Identifying the concepts contained in outcome measures of clinical trials on breast cancer using the International Classification of functioning, Disability and Health as a reference. J Rehabil Med 2004; 44(Suppl): 43-48.

20 Brockow T, Wohlfahrt K, Hillert A, Gehy S, Weigl M, Franke T et al. Identifying the concepts contained in outcome measures of clinical trials on depressive disorders using the International Classification of functioning, Disability and Health as a reference. J Rehabil Med 2004; 44(Suppl): 49-55.

21 Geyh S, Kurt T, Brockow T, Cieza A, Ewert T, Omar Z et al. Identifying the concepts contained in outcome measures of clinical trials on stroke using the International Classification of functioning, Disability and Health as a reference. J Rehabil Med 2004; 44(Suppl): 56-62.

22 Scheuringer M, Grill E, Boldt C, Mittrach R, Müllner P, Stucki G. Systematic review of measures and their concepts used in published studies focusing on rehabilitation in the acute hospital and in early post-acute rehabilitation facilities. Disabil Rehabil 2005; 27: 419-429. 